\title{
Estudio cualitativo sobre las vivencias del cuidador de Alzheimer: adaptación e impacto
}

\section{Qualitative study on the experiences of the Alzheimer caregiver: adaptation and impact \\ Estudo qualitativo sobre as experiências do cuidador de Alzheimer: adaptação e impacto}

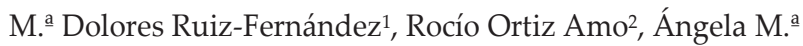 \\ Ortega-Galán ${ }^{3}$ \\ ${ }^{1}$ Departamento de Enfermería, Fisioterapia y Medicina. Universidad de \\ Almería. Almería, España. Correo electrónico: md.ruizfernandez@ual.es \\ ${ }^{2}$ Departamento de Enfermería. Universidad de Huelva. Huelva, España. Correo \\ electrónico:29rocii@gmail.com \\ ${ }^{3}$ Departamento de Enfermería. Universidad de Huelva. Huelva, España. Correo \\ electrónico: amgela.ortega@denf.uhu.es
}

Cómo citar este artículo en edición digital: Ruiz-Fernández, M. ${ }^{a}$ D.; Ortiz Amo, R. E Ortega-Galán, A. M. (2019). Estudio cualitativo sobre las vivencias del cuidador de Alzheimer: adaptación e impacto.

Cultura de los Cuidados (Edición digital), 23(53). Recuperado de http://dx.doi.org/10.14198/cuid.2019.53.03

Correspondencia: Rocío Ortiz Amo2 Avda. Tres de Marzo s/n - 21071 Huelva (España)

Correo electrónico de contacto: 29rocii@gmail.com Recibido: 07/11/2018; Aceptado: 09/01/2019

\section{(c) (i)}

\section{ABSTRACT}

Objective: To deepen, through the experiences of family caregivers, what were the perceptions that caregivers had regarding the process of adaptation of Alzheimer's disease and the impact it had on their lives.

Methodology: Two focus groups of caregivers of patients with Alzheimer-type dementia were formed, representative of the study population.

Results: From the analysis of the discourses five dimensions or units of meaning emerged with fourteen subdimensions. These five dimensions are: knowledge about the illness, the caregiver's suffering, emotions, coping skills and the consequences in the life of the caregiver.

Conclusion: We can determine that the adaptation to the role of caregiver of a person with Alzheimer's is conditioned by the mistaken beliefs or dysfunctional thoughts that arise in the caregiver, the emotions derived from the moral responsibility to assume the role of caregiver from the perspective of gender and, finally, the consequences of care during this period, the repercussions on their personal, family and work life.

Keywords: Alzheimer's disease, family caregivers, experiences, adaptation, experiences. 


\section{RESUMO}

Objetivo: Aprofundar, através das vivências dos familiares cuidadores, quais as percepções que os cuidadores possuíam em relação ao processo de adaptação da doença de Alzheimer e o impacto que isso teve em suas vidas.

Metodologia: Foram constituídos dois grupos focais de cuidadores de pacientes com demência tipo Alzheimer, representativos da população estudada.

Resultados: A partir da análise dos discursos, emergiram cinco dimensões ou unidades de significado com quatorze subdimensões. Essas cinco dimensões são: conhecimento sobre a doença, o sofrimento do cuidador, emoções, habilidades de enfrentamento e as consequências na vida do cuidador.

Conclusão: Podemos determinar que a adaptação ao papel de cuidador de uma pessoa com Alzheimer é condicionada pelas crenças equivocadas ou pensamentos disfuncionais que surgem no cuidador, as emoções derivadas da responsabilidade moral de assumir o papel de cuidador a partir da perspectiva de gênero e, por fim, as consequências do cuidado nesse período, as repercussões na sua vida pessoal, familiar e laboral.

Palavras chave: Doença de Alzheimer, cuidadores familiares, experiências, adaptação, experiências.

\section{RESUMEN}

Objetivo: Profundizar a través de las vivencias de los cuidadores familiares, cuáles eras las percepciones que tenían los cuidadores con respecto al proceso de adaptación de la enfermedad de Alzheimer y el impacto que tenía en sus vidas.

Metodología: Se formaron dos grupos focales de cuidadores de pacientes con demencia tipo Alzheimer, representativos de la población de estudio.

Resultados: Del análisis de los discursos emergieron cinco dimensiones o unidades de significado con catorce subdimensiones. Estas cinco dimensiones son: conocimientos sobre la enfermedad, sufrimiento del cuidador, emociones, capacidad de afrontamiento y las consecuencias en la vida del cuidador.

Conclusión: Podemos determinar que la adaptación al rol de cuidador de una persona con Alzheimer queda condicionada por las creencias erróneas o pensamientos disfuncionales que surgen en el cuidador, las emociones derivadas de la responsabilidad moral de asumir el rol de cuidador desde la perspectiva de género $y$, por último, las consecuencias que produce el cuidado en este periodo las repercusiones en su vida personal, familiar y laboral.

Palabras clave: Enfermedad de Alzheimer, cuidadores familiares, vivencias, adaptación, experiencias.

\section{INTRODUCCIÓN}

La enfermedad de Alzheimer constituye la primera causa de demencia en los países desarrollados, considerándose una enfermedad devastadora tanto para las víctimas, como para los familiares (Organización Mundial de la Salud, 2012). Una de las características de la enfermedad es que se trata de una patología neurodegenerativa compleja, de larga duración y con una sintomatología muy cambiante (Masters, Morris, y Roe, 2015). La mayoría de las personas afectadas presentan síntomas neuropsiquiátricos, llegando a niveles elevados de dependencia $y$ complejidad (Storti, Quintino, Silva, 
Kusumota, y Marques, 2016).

En concreto, la atención y el cuidado de los enfermos lo asume la familia, y dentro del medio familiar, la responsabilidad mayor la ocupa el cuidador principal (Lozano y Huertas, 2017). Investigaciones realizadas en nuestro país determinan que el perfil del cuidador principal es el de mujer con una media de unos 55 años, con bajo nivel cultural, siendo las hijas normalmente las que cuidan de estos enfermos (GarzónMaldonado et al., 2017). El proceso de ser cuidador de una persona diagnosticada con la enfermedad de Alzheimer no es una tarea fácil y tampoco es una situación que surja de forma imprevista, sino que tiene su proceso de adaptación con el trascurso de la enfermedad (Moreno-Cámara, PalominoMoral, Moral-Fernández, Frías-Osuna, y del-Pino-Casado, 2016). Según, el modelo biopsicosocial de estrés de Lazarus y Folkman (1986), el cuidador se enfrenta a una serie de estresores o demandas que en sí mismas no son perjudiciales, lo importante es la evaluación subjetiva que hace el sujeto de la situación y de los recursos para hacerles frente, lo que va a condicionar la adaptación a esta nueva etapa de la vida y las repercusiones que van a producir.

El estilo de afrontamiento de los cuidadores va cambiando con el progreso de la enfermedad. En las fases más iniciales de la enfermedad de su familiar, los cuidadores se inclinan por la utilización de estrategias de afrontamiento más centradas en el abordaje directo del problema, y posteriormente, en fases más avanzadas de la enfermedad modificarse a un estilo más emocional (Ruiz, Gázquez, y Pérez-Fuentes, 2013).

Por otra parte, las consecuencias derivadas del cuidado de sus familiares trascienden a su vida familiar y laboral. El tiempo que dedican al cuidado es cada vez mayor, debido a que la persona cuidada se vuelve más dependiente del cuidador y este se vuelca en satisfacer las necesidades de su familiar, disminuyendo su espacio de ocio, al encontrarse con falta de tiempo. El tiempo que dedican al resto de su familia también se ve reducido, lo que repercute en un aumento de conflictos familiares y aislamiento social (Uribe et al., 2017). Así pues, el objetivo de este estudio consistió en profundizar a través de las vivencias de los cuidadores familiares, cuáles eras las percepciones que tenían los cuidadores con respecto al proceso de adaptación de la enfermedad de Alzheimer y el impacto que producía este proceso en sus vidas.

\section{METODOLOGÍA}

\section{Diseño}

Diseño de investigación cualitativa con un enfoque fenomenológico hermenéutico de acuerdo al modelo de Van Manen (2003). Esta perspectiva, nos permitió profundizar en las experiencias de los cuidadores familiares sobre los aspectos más importantes vividos en el proceso de adaptación del cuidado de un enfermo diagnosticado de Alzheimer, y el significado que había supuesto para ellos el nuevo rol de cuidador.

\section{Participantes}

En total participaron 14 cuidadores. Se formaron dos grupos focales de cuidadores principales. Como criterios de inclusión seleccionamos a cuidadores familiares de pacientes diagnosticados de demencia tipo Alzheimer, incluidos en el proceso de atención sanitaria a demencias. Excluimos a aquellos cuidadores que no cumplían con estos criterios, es decir, que no fueran cuidadores familiares, ni principales, o que su familiar padeciera otro tipo de demencia. 


\section{Selección de los participantes}

La población seleccionada para la participación en los grupos focales se obtuvo, de las historias de salud del programa informático utilizado en los centros sanitarios y de la base de datos de cuidadores familiares que tenían la tarjeta más cuidado, dentro del Plan de Atención a Cuidadores Familiares del Servicio Andaluz de Salud. Uno de los requisitos previos a la formación de los grupos, consistió en que los cuidadores no se conocieran entre sí, para evitar sesgos en la dinámica y el desarrollo de los grupos.

\section{Recogida de datos}

Las sesiones tuvieron una duración entre 60 y 90 minutos, en salas habilitadas en los centros sanitarios cercanos a la población de estudio. Se localizó a los integrantes de los grupos por contacto telefónico o a través de informantes claves, proporcionándoles en todo momento la asistencia a la reunión en un horario adecuado y cercano a su residencia habitual, con el objeto de facilitar su asistencia. Se estableció un guion de preguntas para garantizar en todo momento la recogida de toda la información necesaria para conseguir el objetivo del estudio (tabla 1). El investigador principal dirigió cada una las sesiones como moderador del grupo y un observador, tomó las anotaciones oportunas en una hoja de registro. Se grabó el desarrollo de la sesión en formato audio para su posterior transcripción y análisis, siempre con el consentimiento informado previo de forma oral y por escrito de los asistentes. Las sesiones en cada uno de los grupos focales se desarrollaron en las siguientes fases: introducción, preparación del grupo, debate a fondo y clausura del grupo (García-Calvente y Mateo, 2000).

TABLA 1: Guion de preguntas: grupo focal

\begin{tabular}{lcccc}
\hline $\begin{array}{c}\text { Participantes } \\
(\mathbf{P})\end{array}$ & Edad & Sexo & Grado parentesco & $\begin{array}{c}\text { Tiempo de } \\
\text { cuidado (años) }\end{array}$ \\
\hline 1 & 56 & M & Nuera & 5 \\
\hline 2 & 49 & M & Hija & 4 \\
\hline 3 & 49 & M & Hija & 16 \\
\hline 4 & 61 & M & Hija & 10 \\
\hline 5 & 72 & M & Cónyuge & 7 \\
\hline 6 & 48 & M & Hija & 2 \\
\hline 7 & 51 & M & Hija & 3 \\
\hline 8 & 52 & M & Nuera & 5 \\
\hline 9 & 41 & M & Nieta & 4 \\
\hline 10 & 51 & M & Nuera & 2 \\
\hline 11 & 55 & M & Cónyuge & 3 \\
\hline 12 & 47 & M & Hija & 2 \\
\hline 13 & 65 & M & Hija & 4 \\
\hline 14 & 49 & M & Hija & 6 \\
\hline
\end{tabular}

\section{Análisis de los datos}

Para el análisis de la información de los discursos de los grupos focales se ha seguido la propuesta desarrollada por Giorgi (1997) que consta de una serie de etapas: En un primer lugar, y tras la trascripción literal de 


\section{Cultura de los Cuidados}

los discursos se realizó una lectura en profundidad. En segundo lugar, se procedió a otra lectura y posteriormente, se extrajeron todas aquellas unidades de significado más relevantes que emergían de los discursos. Una vez identificadas todas las unidades de significado, estas se reagruparon en categorías o dimensiones más generales que aglutinaban a aquellas unidades de significado más pequeñas y que tenían algo en común. Como última etapa, se interpretaron los contenidos de cada una de las categorías y dimensiones analizadas de acuerdo al fenómeno o experiencia vivida por varios investigadores expertos en el tema. Como soporte informático del análisis de los discursos de las entrevistas y con el fin de organizar las categorías, y obtener los nodos correspondientes, se utilizó el software informático Atlas ti versión 6.0.

\section{Consideraciones éticas}

La investigación ha obtenido el dictamen favorable del comité de ética de la investigación correspondiente a la provincia donde se ha realizado el estudio. A los participantes se les informó de forma verbal y por escrito del propósito del estudio, obteniendo su consentimiento informado en un documento diseñado para tal fin. En el trascurso de la investigación se ha mantenido la confidencialidad y el anonimato de los participantes, respetando los principios bioéticos de la declaración de Helsinki. Los datos de los discursos han sido custodiados y protegidos, cumpliendo la normativa que se encuentra vigente en nuestro país en cuanto a la protección oficial de datos de carácter personal.

\section{RESULTADOS}

La muestra estuvo compuesta por 14 participantes con una edad media de 53,2 años. En la tabla 2, se describen las principales características sociodemográficas de los participantes.

TABLA 2: Características sociodemográficas de los participantes

\begin{tabular}{lllcc}
\hline $\begin{array}{c}\text { Participantes } \\
(\mathbf{P})\end{array}$ & Edad & Sexo & Grado parentesco & $\begin{array}{c}\text { Tiempo de } \\
\text { cuidado (años) }\end{array}$ \\
\hline 1 & 56 & M & Nuera & 5 \\
\hline 2 & 49 & M & Hija & 4 \\
\hline 3 & 49 & M & Hija & 16 \\
\hline 4 & 61 & M & Hija & 10 \\
\hline 5 & 72 & M & Cónyuge & 7 \\
\hline 6 & 48 & M & Hija & 2 \\
\hline 7 & 51 & M & Hija & 3 \\
\hline 8 & 52 & M & Nuera & 5 \\
\hline 9 & 41 & M & Nieta & 4 \\
\hline 10 & 51 & M & Nuera & 2 \\
\hline 11 & 55 & M & Cónyuge & 3 \\
\hline 12 & 47 & M & Hija & 2 \\
\hline 13 & 65 & M & Hija & 4 \\
\hline 14 & 49 & M & Hija & 6 \\
\hline
\end{tabular}




\section{Cultura de los Cuidados}

En el análisis de los discursos emergieron 5 dimensiones o unidades de significado con 14 subdimensiones, englobadas todas estas en una categoría o familia más amplia relacionada con el proceso de adaptación e impacto en la vida de un cuidador de un enfermo de Alzheimer tal y como puede observarse en la tabla 3. Estas cinco dimensiones son: conocimientos sobre la enfermedad, sufrimiento del cuidador, emociones, capacidad de afrontamiento y las consecuencias en la vida del cuidador.

TABLA 3: El proceso de adaptación e impacto: dimensiones y subdimensiones

\begin{tabular}{|c|c|}
\hline Dimensiones & Subdimensiones \\
\hline \multirow{3}{*}{$\begin{array}{l}\text { Conocimientos sobre la } \\
\text { enfermedad }\end{array}$} & Creencias con respecto a la enfermedad \\
\hline & Escasa información por parte de los profesionales \\
\hline & Miedo que genera el desconocimiento \\
\hline \multirow{4}{*}{ Sufrimiento del cuidador } & Sufrimiento del cuidador por no reconocer a su familiar \\
\hline & $\begin{array}{l}\text { Sufrimiento del cuidador por no ser reconocido por su } \\
\text { familiar }\end{array}$ \\
\hline & Sufrimiento del cuidador hacia otros familiares \\
\hline & Sufrimiento ante la pérdida de su familiar \\
\hline \multirow{5}{*}{ Emociones } & Sentimiento de aislamiento \\
\hline & Obligación de la mujer de asumir el papel \\
\hline & Sentimiento de responsabilidad \\
\hline & Sentimiento de resignación \\
\hline & Agotamiento \\
\hline Capacidad de afrontamiento & Tranquilidad hacia el cuidado \\
\hline \multirow{4}{*}{$\begin{array}{l}\text { Consecuencias en la vida del } \\
\text { cuidador }\end{array}$} & Cambio de vida personal \\
\hline & Modificación en relaciones sociales \\
\hline & Modificación en su vida familiar e inversión de roles \\
\hline & Limitación de tiempo \\
\hline
\end{tabular}

\section{Conocimientos sobre la enfermedad}

a) Creencias con respecto a la enfermedad.

Las cuidadoras nos relatan la interiorización de ciertas creencias con respecto a la enfermedad de Alzheimer que surgen como un mecanismo para comprender la enfermedad. Esta forma de percibir la enfermedad tiene un sentido, les permite sobrellevar una enfermedad que debuta con una sintomatología tan variada y compleja. El asumir ciertas creencias lleva implícito para las cuidadoras, una explicación del comportamiento de su familiar y un mayor afrontamiento de la enfermedad.

"Yo creo que debe de haber varias etapas, es decir, debe de haber varias clases de enfermedades, siendo la misma, que avanza de diferentes maneras, unas más rápida que otras". P1

"Si, hay una sustancia que se pega a las neuronas, la que causa la enfermedad". P9 


\section{b) Escasa información por parte de los profesionales.}

El desconocimiento sobre la enfermedad y todos los aspectos relacionados con esta en cuestiones, como el tratamiento que reciben los familiares o la información adecuada acerca del diagnóstico, proviene en ocasiones de la escasez de información que proporcionan los profesionales sanitarios a los cuidadores. Esta escasez de información, genera un nivel de incertidumbre que obliga a las cuidadoras a recurrir a ciertos recursos personales y creencias internas, que les permitan dar sentido a lo que están viviendo.

"Si la enfermedad, no me han dicho exactamente lo que tiene, sé qué es Alzheimer porque se lo conté a su médico de familia, le dije a su médico que se le olvidaban las cosas". P1

"Yo a mi madre, la he encontrado distinta desde hace años. Se lo decía a su médico, pero me decía que no era nada. Su médico me lo dijo hace poco. Yo le preguntaba si era Alzheimer, me decía bueno, esto cuesta al principio. Pero, yo lo sabía antes de que me lo dijera". P6

\section{c) Miedo que genera el desconocimiento.}

En el cuidador surgen temores y miedos con respecto a la enfermedad en sí, y a la progresión de esta. Estos temores están fundamentados en experiencias previas de cuidado o de familiares allegados y a su vez, reforzados por la escasez de información. Los temores nacen por el desconocimiento y a su vez por ciertas creencias relacionadas con la naturaleza de la enfermedad, la evolución de la misma, las fases e incluso a ciertos comportamientos. El componente hereditario es otro factor que origina ciertos temores en las cuidadoras.

"Es una enfermedad, que me da mucho miedo, le temo porque he visto varios casos". P1

"Le temo a la fase de mi abuela, estaba muy rebelde quería irse a su casa, quería cerrar las puertas., yo le temo a que mi madre llegue a esa fase, me da miedo a que se encierre". P4

\section{Sufrimiento del cuidador}

a) Sufrimiento del cuidador por no reconocer a su familiar.

El sufrimiento que padecen las cuidadoras con respecto al no reconocimiento de su familiar después de la enfermedad, queda reflejado en los discursos. Surge un dolor inmenso y una pena con respecto a la pérdida de su familiar, manifestado por el anhelo y el vacío que sienten al no reconocer a su familiar como consecuencia de las secuelas de la enfermedad.

"Me cuesta mucho asumirlo. Lo que era mi madre y como está ahora, a pesar de que todavía no está muy grave. No es mi madre. Entonces a mí, me cuesta mucho eso, porque he visto a mi madre que no es mi madre". P9

"Eso, el mundo encima., yo por lo menos, jno tengo a mi madre, no es ella, tengo un vacío enorme!". P8

\section{b) Sufrimiento del cuidador por no ser reconocido por su familiar.}

Por otra parte, el sufrimiento que sienten las cuidadoras viene determinado por el no reconocimiento de la cuidadora por su familiar más allegado. Esta situación genera en la cuidadora un inmenso dolor $y$ sufrimiento, puesto que para las cuidadoras es muy importante el reconocimiento de sus familiares, sintiéndose aterrados ante esta vivencia.

"Llega un momento, en que no te conoce y no quiere que le toques, se piensa que eres algo extraño". P4

"A mí me miedo, que llegue el momento de que no me reconozca. Es lo que más pánico me da". P4 
c) Sufrimiento del cuidador hacia otros familiares.

También se hace presente el sufrimiento y dolor hacia otros familiares que conviven con él, como pueden ser los cónyuges en aquellas circunstancias en que las cuidadoras son hijos o hijas. Las cuidadoras sufren de ver la negación y la no aceptación de la enfermedad en estos familiares, con respecto a su pareja enferma. Incluso, el comunicar el diagnostico a sus familiares cercanos, puede ocasionar tal angustia y desesperanza en las cuidadoras que desemboca en el sentimiento de la compasión y la necesidad de empatizar.

"Igual que he dicho antes, me afecta mi madre. Mi madre tiene 68 años y mi padre 88. Hoy estaba tembloroso, le hemos tenido que dar de comer, mi madre estaba nerviosa, no acepta la situación de mi padre, no ve que va a peor y me afecta ver a mi madre, como no es consciente de que mi padre va a peor. Me parte el corazón, el sufrimiento de mi madre, porque mi padre no es consciente". P2

"Yo la verdad es que cuando me lo dijeron, me quedé tranquila, no reaccioné, me lo esperaba, aunque pensaba también en un tumor. Unos tíos míos, murieron de la misma enfermedad. Sufría por mi madre que la tenía al lado, pero observé que no sabía lo que decía el médico y sufría con pensar, en cómo decirle a mi madre, lo que tenía mi padre. Mi madre está enferma y siempre ha sido muy fuerte, siempre ha llevado la casa". P2

\section{d) Sufrimiento ante la pérdida de su familiar.}

El sufrimiento del cuidador puede llegar a límites inalcanzables, inclusive hasta anticipar la pérdida de su familiar. La cuidadora llega a cuestionarse la situación emocional que puede desencadenar su ausencia, generando incertidumbre en un futuro próximo.
"Yo pienso, aún en lo peor. ¿Cómo me quedaré, cuando mi madre no esté? Después de 18 años". P3

"Yo se lo digo a mi hermana, que sufre antes de tiempo". P10

\section{Emociones}

En esta categoría se describen las emociones que perciben las cuidadoras cuando asumen el rol de cuidador.

a) Sentimiento de aislamiento.

Las cuidadoras perciben las limitaciones en las relaciones sociales y comienzan a sentirse aislados y en soledad.

"Yo no puedo quedar con nadie, ¿para qué? ¿Para no estar a gusto? Prefiero estar en la casa". P2

"Yo estoy por ahí, tomando café, se pasa la hora y me dan los nervios, ya llega la hora". P3

\section{b) Obligación de la mujer de asumir el papel.}

Surge la obligación moral de asumir el rol de cuidador. Perciben las desigualdades de género y el deber de la mujer, asociado a todos los valores contextuales y socioculturales tradicionales de sucesivas generaciones en el pasado.

"No es lo mismo, una hija que cuida a su madre, que otra que no. Esa tranquilidad, ¿y el vacío que se queda?". P2

"Mi hija se ha adaptado., yo tengo a mi hija que me ayuda mucho, pero como son los niños, son diferentes a las niñas, pasan de la abuela y de la madre". P4

\section{c) Sentimiento de responsabilidad.}

Otro de los sentimientos que perciben las cuidadoras es el sentimiento de responsabilidad y no poder ser sustituido por otro miembro de la familia. Las cuidadoras asumen toda la responsabilidad, a pesar de tener apoyo familiar. 
"Tengo a mi cuñada, a mi hermano y a mi marido, me apoyan bastante. Lo que pasa, es que ¿cómo les dejas el cargo a los demás? Es un cargo, muy gordo". P2

"Si tengo que ir a un médico deprisa, si, están mis hijos, pero la carga la llevo yo. Solamente para casos muy urgentes, que lo necesito". P8

\section{d) Sentimiento de resignación.}

El sentimiento de resignación emerge en los discursos, las cuidadoras perciben la obligación de asumir el papel de cuidador, cuando no existe una ocupación laboral o no se encuentran en situación laboral activa.

"Tengo a mi hermana, lo que pasa es que mi hermana está trabajando. Mi hermana no va a dejar el trabajo mientras yo pueda, su trabajo es muy bonito". P4

\section{e) Agotamiento.}

La sensación de sentirse agotada física y psíquica comienza a emerger en las cuidadoras, como consecuencia del sentimiento de responsabilidad y la obligación que tienen.

"No me siento bien, veces me siento agotada, son dos personas". P2

"Yo me siento unos días bien y otros días mal. Unos días se siente uno más agobiada y otros días, menos". P10

\section{Capacidad de afrontamiento}

En las cuidadoras esta virtud la perciben como la capacidad del cuidador de poder afrontar las situaciones más problemáticas de su familiar, incluyendo el conocimiento de la persona cuidada desde una perspectiva holística y en dónde el conocimiento psicológico es fundamental.

"Soy muy tranquila, relajo mucho a la persona y al enfermo que cuido. Ahora he tenido una experiencia desagradable y a mi tía le he ayudado mucho. Es también fundamental tener esa capacidad, aunque por dentro genere deterioro". P4

"Mi marido, tiene que ser yo, no quiere a nadie, como no sea yo. Nadie más que yo, no lo entiende". P8

\section{Consecuencias en la vida del cuidador \\ a) Cambio de vida personal.}

La cuidadora percibe como su vida personal cambia de forma drástica. Este cambio de vida, produce un gran impacto a todos los niveles de su esfera personal, relacionado con el papel de la mujer en el cuidado, puesto que no está instaurado en los roles que puede asumir la mujer de hoy.

"Es que las personas mayores, han tenido otra vida diferente a la nuestra, están adaptados a esa forma de vivir. Pero, es que nosotros nos tenemos que adaptar a otra vida. Voy a ver cómo me explico, las personas mayores, como mi madre, han nacido para casarse, tener hijos, cuidar a las suegras, a los padres, al marido". P3

\section{b) Modificación en relaciones sociales.}

Los cambios en las relaciones sociales son evidentes y son percibidos por los cuidadores que comienzan a sentirse mal. Abandonan las actividades lúdicas, no tienen tiempo y comienzan a aislarse.

"Muy mal, he dejado la gimnasia, los talleres de cocina, mi pintura. Me siento muy mal. Antes acudía a los talleres, por las tardes. Ahora no puedo". P3

"Yo no puedo salir a la calle, ni veo telediarios, ni puedo hablarle, no puedo ir a la playa. Siempre estoy encerrada". P8

\section{c) Modificación en su vida familiar e inversión de roles.}

El cambio tan grande que produce el cuidado en su vida familiar, unido al cambio de roles entre padres e hijos, produce un 
sentimiento de sobrecarga y de agobio en el cuidador. Comienza a sentirse tan indispensable en su familia como también al cuidado de sus padres, que se convierten en personas dependientes y a su cargo.

"Cuando mi padre ha estado ingresado, yo me he quedado con él por las noches. Como cambia tanto mi vida, con mi casa y eso que mis hijos son grandes. Me he dado cuenta, de que hago tanta falta en mi casa, como también en la casa de mis padres. Entonces, como mi vida cambia tanto y mi vida da un giro tan grande, ese es el agobio que me da". P9

\section{d) Limitación de tiempo.}

La falta de tiempo es otra de las limitaciones que aparecen en los discursos de los cuidadores y que repercute en su vida personal. El no poder compatibilizar las actividades de la vida cotidiana y las tareas propias del cuidador, les preocupa y les genera un malestar emocional.

"Te falta tiempo para hacer las cosas de tu casa, tienes que hacer la comida, estar pendiente de ella". P7

"No puedo ir a ningún sitio, porque por las mañanas voy desesperada a comprar, pensando en que se puede caer mi madre". P3

\section{DISCUSIÓN}

En este estudio podemos ver cómo han vivido el proceso de adaptación los cuidadores familiares de enfermos con Alzheimer, al igual que se han descrito los sentimientos y emociones que han surgido en el cuidador en este periodo, y como ha sido el impacto en su vida personal, familiar y social. En general, los datos obtenidos en nuestro estudio acerca de las características sociodemográficas de los cuidadores familiares de pacientes con demencia tipo Alzheimer, nos muestran un perfil de población muy similar a la obtenida en otros estudios nacionales con este tipo de cuidadores. Así pues, partimos de una población de mediana edad, con una edad comprendida entre 50-60 años, mayormente mujeres en comparación de hombres. En cuanto a la relación de parentesco con el familiar cuidado, principalmente son hijas y posteriormente los cónyuges (GarzónMaldonado et al., 2017).

La formulación de ciertas creencias disfuncionales y su interiorización con respecto a la enfermedad de Alzheimer, les permiten a los cuidadores comprender la enfermedad y el comportamiento de su familiar, lo que lleva implícito un mayor afrontamiento de la enfermedad. Un aspecto muy importante relacionado con las creencias internas, es la escasez de conocimiento que tienen estos cuidadores (Fauth, Femia, y Zarit, 2016) y que está relacionado frecuentemente con el déficit de información que proporcionan los profesionales con respecto al diagnóstico de la enfermedad; esto puede producir una serie de miedos y temores que pueden desembocar en estrés y sobrecarga en el cuidador, tal y como lo describe Abreu et al. (2018).

Una característica de los cuidadores de Alzheimer y que se ha observado también en nuestro estudio, al igual que otras investigaciones, es la sensación del cuidador de estar presente y de ser la única persona que puede ofrecer los cuidados (Kimura et al., 2018). Este sentimiento de ser la única persona que puede ofrecer los cuidados, está motivado por el instinto de la compasión que surge ante el sufrimiento del cuidador por la pérdida de su familiar, así como, el que su familiar no lo reconozca. Masters, Morris y Roe (2015) en su investigación observo que los cuidadores que sentían un amor más compasivo por sus parejas con 
enfermedad de Alzheimer, tenían menos carga de cuidado y su rol de cuidador era más positivo, que aquellos cuidadores que sintieron menos amor compasivo por sus parejas con la enfermedad. Por otro lado, hemos observado el sufrimiento del cuidador ante una pérdida inminente de su familiar y la elaboración del duelo anticipado, como un proceso de adaptación que deben de superar los cuidadores y que necesitan elaborar puesto que les permite la aceptación de vivir con un familiar enfermo y además anticipar la muerte de su ser querido (Leiva-Díaz, Hernández-Rojas, y Aguirre-Mora, 2016).

La responsabilidad y la obligación moral de asumir el cuidado por la mujer ha surgido en nuestra investigación desde el comienzo de la enfermedad de su familiar, en línea con otros estudios en los que los valores otorgados al papel del cuidador implican desigualdad de género y el condicionamiento de la mujer para ocupar esta tarea. (Garzón-Maldonado et al., 2017). Podemos argumentar que se puede asumir esta responsabilidad desde la afectividad hacia un miembro de la familia y dentro de una actitud conservadora que ha existido a lo largo de la historia con un enfoque de género, sin ninguna razón en particular, o simplemente porque no existe disponibilidad de otras personas o ayuda formal para realizarlo $\mathrm{y}$, por tanto, la mujer es la persona ideal para realizar este trabajo (Marcos et al., 2015).

Por otra parte, comienza a ser evidente al igual que ocurre en otros cuidadores, la pérdida de las relaciones sociales, el aislamiento, y el agotamiento físico y psíquico en los cuidadores, unido al impacto en diferentes esferas de su vida personal, familiar y laboral, tal y como se ha evidenciado en otros estudios (MorenoCámara et al., 2016). Aun así, se sienten competentes y conocen los procedimientos básicos para el cuidado y para el manejo de estos enfermos, virtud muy valorada por los cuidadores. (Storti, Quintino, Silva, Kusumota, y Marques, 2016).

Una de las principales limitaciones del estudio han sido las propias características de los cuidadores familiares, al ser todas mujeres y no haber incluido ningún cuidador hombre, quizás hayamos podido solo visualizar el proceso de adaptación que sufre el género femenino. Por tanto, sería conveniente incluir en próximos estudios la perspectiva de cuidador hombre, con el fin de visualizar ambas vertientes. Este trabajo debe de servir de base para generar nuevas hipótesis $\mathrm{y}$, por lo tanto, nuevas líneas de investigación futuras, donde se incluya la perspectiva del género masculino como cuidador principal; esto nos permitirá conocer otra visión por parte de los cuidadores; para así seguir avanzando en mejorar la calidad de vida y el bienestar de los cuidadores de pacientes con demencia tipo Alzheimer.

\section{CONCLUSIONES}

A modo de conclusión, podemos determinar que la adaptación al rol de cuidador de una persona con Alzheimer queda condicionada por las creencias erróneas o pensamientos disfuncionales que surgen en el cuidador, muchas veces a consecuencia de la escasez de información que ofrecen los profesionales sanitarios. Por otra parte, aparece el cuidado compasivo ante el sufrimiento del cuidador por las repercusiones de la enfermedad en la persona querida, además de, las emociones derivadas de la responsabilidad y obligaciones morales de asumir el rol de cuidador desde la perspectiva de género: En todo este periodo de adaptación, la 
competencia tiene un valor prioritario para poder afrontar la situación que vive el cuidador. Y, por último, enfatizar el gran impacto que produce el cuidado en este periodo las repercusiones en su vida personal, familiar y laboral. Así pues, es prioritario diseñar y aplicar programas de intervención en estos cuidadores, desde el comienzo de la enfermedad, de tal modo que nos permitan prevenir la sobrecarga de los cuidadores y en dónde, los profesionales de enfermería tenemos una gran labor en el ámbito comunitario.

\section{REFERENCIAS}

- Abreu, W., Tolson, D., Jackson, G., y Costa, N. (2018). A cross-sectional study of family caregiver burden and psychological distress linked to frailty and functional dependency of a relative with advanced dementia. Dementia, 0(0), 1-18.

- Fauth, E.B., Femia, E.E., y Zarit, S.H. (2016). Resistiveness to Care during Assistance with Activities of Daily Living in Non-institutionalized Persons with Dementia: Associations with Informal Caregivers' Stress and Well-being. Aging $\mathcal{E}$ Mental Health, 20(9), 888-898.

- García-Calvente, M., y Mateo, I. (2000). El grupo focal como técnica de investigación cualitativa en salud: diseño y puesta en práctica. Atención primaria, 25(3), 181-186.

- Garzón-Maldonado, F.J., Gutiérrez-Bedmar, M., García-Casares, N., Pérez-Errázquin, F., GallardoTur, A., y Martínez-Valle Torres, M.D. (2017). Healthrelated quality of life in caregivers of patients with Alzheimer disease. Neurología, 32 (8), 508-515.

- Giorgi, A. (1997). The theory, practice, and evaluation of the phenomenological method as a qualitative research procedure. Journal of Phenomenological Psychology, 28(2), 235-260.

- Kimura, N. R., Baptista, M. A. T., Santos, R. L., Portugal, M. D. G., Johannenssen, A., Barca, M. L., y Dourado, M. C. (2018). Caregivers' Perspectives of Quality of Life of People with Young-and Late-Onset Alzheimer Disease. Journal of geriatric psychiatry and neurology, 31(2), 76-83.

- Uribe, F. L., Gräske, J., Grill, S., Heinrich, S., SchäferWalkmann, S., Thyrian, J. R. \& Holle, B. (2017). Regional dementia care networks in Germany: changes in caregiver burden at one-year follow-up and associated factors. International psychogeriatrics, 29(6), 991-1004.

- Lazarus, R.S., y Folkman, S. (1986). Estrés y procesos cognitivos. Barcelona: Martínez Roca.

- Leiva-Díaz, V., Hernández-Rojas, M.E., y AguirreMora, E. (2016). Experiencias de familias que conviven con una persona con diagnóstico de Alzheimer. Enfermería Actual de Costa Rica, 30, 1-22.

- Lozano-López, B., y Huertas-Pérez, M.M. (2017). Cuidados paliativos, cuidados compartidos. Cultura de los Cuidados (Edición digital), 21(49), 100-107. Recuperado de http://dx.doi.org/10.14198/cuid.2017.49.11.

- Marcos, M.M., y de la Cuesta, C. (2015). La experiencia del cuidado de las mujeres cuidadoras con procesos crónicos de salud de familiares dependientes. Atención Primaria, 48(2), 77-84.

- Masters, M., Morris, J., y Roe, C. (2015). Noncognitive symptoms of early Alzheimer disease. Neurology, 84(6), 617-622.

- Moreno-Cámara, S., Palomino-Moral, P. Á., MoralFernández, L., Frías-Osuna, A., y del Pino-Casado, R. (2016). Problemas en el proceso de adaptación a los cambios en personas cuidadoras familiares de mayores con demencia. Gaceta Sanitaria, 30(3), 201207.

- Organización Mundial de la Salud (OMS). (2012). Dementia: a public health priority. Alzheimer's disease International. United Kingdom: OMS.

- Ruiz, M.D., Gázquez, J.J., y Pérez-Fuentes, M.C. (2013). Estrategias de afrontamiento en cuidadores familiares de enfermos de Alzheimer. En J. J. Gázquez, M. C. Pérez-Fuentes, M. M. Molero y I. Mercader (Eds.), Calidad de vida, cuidadores e intervención para la mejora de la salud en el envejecimiento (Volumen I) (pp. 221-226). Almería: ASUNIVEP.

- Storti, L.B., Quintino, D.T., Silva, N.M., Kusumota, L., y Marques, S. (2016). Neuropsychiatric symptoms of the elderly with Alzheimer's disease and the family caregivers' distress. Revista Latino-americana de Enfermagem, 24, e2751.

- Van Manen, M. (2003). Investigación educativa y experiencia vivida: Ciencia humana para una pedagogía de la acción y la sensibilidad. Barcelona: Idea Books. 